\title{
The effect of bisphosphonate discontinuation on the incidence of postoperative medication-related osteonecrosis of the jaw after tooth extraction
}

\author{
Sang-Hoon Kang, Se-Jin Park, Moon-Key Kim \\ Department of Oral and Maxillofacial Surgery, National Health Insurance Service Ilsan Hospital, Goyang, Korea
}

\begin{abstract}
J Korean Assoc Oral Maxillofac Surg 2020;46:78-83)
Objectives: The discontinuation of bisphosphonate (BP) treatment before tooth extraction may induce medication-related osteonecrosis of the jaw (MRONJ). Whether the long-term discontinuation of BP treatment before tooth extraction affects the risk of developing MRONJ after tooth extraction or whether extended drug holidays induce systemic side effects remains unclear. The present study assessed the incidence of MRONJ among patients who underwent tooth extraction and did not discontinue BP therapy prior to the procedure.

Materials and Methods: Patients were classified according to whether or not they discontinued BP therapy before tooth extraction. Differences in the incidence of MRONJ after tooth extraction were compared between the two groups using the chi-squared test.

Results: The BP-continuation (BPC) and BP-discontinuation (BPDC) groups included 179 and 286 patients, respectively. One patient in the BPC group and no patients in the BPDC group developed MRONJ $(P=0.385)$. The patients in the BPDC group stopped receiving BP therapy at a mean of $39.0 \pm 35.5$ months prior to tooth extraction.

Conclusion: The possibility of pre-existing MRONJ in the extraction area must be considered during the extraction procedure. Routine discontinuation of BP medications for several months before the extraction procedure should be carefully considered, as evidence of its efficacy in reducing the development of post-extraction MRONJ is limited.
\end{abstract}

Key words: Bisphosphonate, Medication-related osteonecrosis of the jaw, Tooth extraction, Discontinuation

[paper submitted 2019. 7. 15 / revised 2019. 9. 2 / accepted 2019. 9. 11]

\section{Introduction}

Though the use of bisphosphonate (BP) to inhibit bony resorption and other antiresorptive or antiangiogenic agents during dental procedures is widespread, it can induce medication-related osteonecrosis of the jaw (MRONJ). MRONJ is most frequently observed following tooth extractions or minor dental surgery ${ }^{2}$ - with the former procedure being one of the most common in the field of dentistry. The mechanism underlying the onset of MRONJ after tooth extraction may

\footnotetext{
Sang-Hoon Kang

Department of Oral and Maxillofacial Surgery, National Health Insurance Service Ilsan Hospital, 100 Ilsan-ro, Ilsan-donggu, Goyang 10444, Korea TEL: +82-31-900-0267 FAX: +82-303-3448-7139

E-mail: omskang@nhimc.or.kr

ORCID: https://orcid.org/0000-0003-3335-3040

(c) This is an open-access article distributed under the terms of the Creative Commons Attribution Non-Commercial License (http://creativecommons.org/ licenses/by-nc/4.0/), which permits unrestricted non-commercial use, distribution, and reproduction in any medium, provided the original work is properly cited. Copyright (C) 2020 The Korean Association of Oral and Maxillofacial Surgeons. All rights reserved.
}

involve trauma to the alveolar bone during the procedure as well as complications arising during recovery ${ }^{3-5}$.

Preventive dental treatment should be conducted to preclude the need for invasive dental treatment and BP therapy ${ }^{6}$. Previous research has attempted to devise preventative methods that can be used during dental procedures and BP therapy to reduce the incidence of $\mathrm{MRONJ}^{7}$. A BP drug holiday prior to invasive dental treatment was also suggested as a means of reducing the serum level of free BP and decreasing the incidence of $\mathrm{MRONJ}^{8,9}$. However, the incidence of MRONJ after tooth extraction is relatively low among patients exposed to oral $\mathrm{BPs}^{10,11}$, and a short-term drug holiday may not effectively decrease the incidence of MRONJ after tooth extraction $^{12,13}$.

A complete protocol has not been developed for the discontinuation of BP treatment before tooth extraction'. Whether the long-term discontinuation of BP treatment before tooth extraction affects the risk of developing MRONJ after the extraction or whether systemic side effects occur as the result of 
the long-term drug holiday remains unclear ${ }^{12-15}$.

The present study assessed the incidence of MRONJ in patients who underwent tooth extraction, had not discontinued BP therapy prior to the procedure, and were determined to not have MRONJ at the extraction site prior to surgery.

\section{Materials and Methods}

In the present non-randomized, single-center, retrospective cohort study, we analyzed the medical records of patients who were treated with $\mathrm{BP}$ and underwent tooth extraction between March 1, 2008 and December 31, 2017. Records that lacked information concerning the medication regimen and its duration, even if they were reported by the patient, were excluded from the study. Only cases in which BP was formally prescribed by our hospital and we could access complete prescription records were included. All the patients included in the study received BP medication prior to tooth extraction. None of the patients had undergone radiation therapy. Patients whose preoperative symptoms indicated the presence of MRONJ as defined by the American Association of Oral and Maxillofacial Surgeons guidelines were excluded ${ }^{9}$. The study also excluded patients with an unclear diagnosis of MRONJ before tooth extraction who exhibited osteonecrosis during the procedure.

Tooth extraction was performed under local anesthesia. Procedures consisted of the simple removal of the maxillary or mandibular teeth, surgical extractions requiring curettage, and surgical extractions requiring bone removal and root separation. After the extraction procedures, filling material (i.e., bone graft material such as collagen or fibrin) was not applied to the extraction socket. All the patients received oral prophylactic antibiotics 1 hour before the procedure. Patients exhibiting postoperative symptoms indicative of MRONJ were assumed to have developed the condition after the extractions.

All the patients were categorized into one of two groups: $\mathrm{BP}$-continuation (BPC) and BP-discontinuation (BPDC). In the BPC group, all the patients were prescribed BP medication before and up until the tooth extraction procedure. Patients in the BPDC group had received BP medication but discontinued the BP therapy prior to the tooth extraction procedure. The reasons for BP discontinuation were not clearly defined in the medical records. Descriptive statistics were used to determine the effectiveness of discontinuing BP prior to tooth extraction. Patients who took BP for more than 3 years were analyzed separately. All statistical analyses were performed in IBM SPSS Statistics (ver. 23; IBM, Armonk, NY, USA). Significance was set at $P \leq 0.05$. Differences in age and the duration of BP medication were compared by t-tests. Differences in the incidence of MRONJ after tooth extraction, sex, number of extracted teeth according to the maxilla/ mandible and anterior/posterior lesions, and extraction method were compared between the two groups using chi-squared tests.

\section{Results}

The medical records of 465 patients (420 females) for a total of 1,323 extracted teeth were assessed. The mean ages of the male ( \pm standard deviation $[\mathrm{SD}], 63.7 \pm 10.5$ years) and female $(69.3 \pm 8.8$ years $)$ were significantly different $(P<0.001)$. The BPC and BPDC groups included 179 and 286 patients, respectively. The mean age of the BPC group (70.3 \pm 9.2 years) was significantly higher than that of the BPDC group (67.8 \pm 8.9 years; $P=0.003$ ).

With regards to the period of $\mathrm{BP}$ discontinuity, the patients in the BPDC group did not receive BP therapy during 39.0 \pm 35.5 months (mean $\pm \mathrm{SD}$ ) prior to tooth extraction.(Table 1) Concerning the method of BP administration, 149 patients in the BPC group received oral BP, while 260 patients in the BPDC group received oral alendronate sodium $(P=0.040)$. Fifteen patients in both groups received alendronate and intravenous (IV) injections of ibandronate. The mean duration of the medication regimen was significantly different $(P<0.001)$ in the BPC (40.0 \pm 35.6 months) and BPDC (14.0 \pm 16.8 months) groups. Fifteen patients in the BPC and BPDC groups received IV injections of ibandronate for $23.0 \pm 18.7$ months and $13.4 \pm 18.6$ months, respectively $(P=0.171)$.

In the BPC and BPDC groups, 89 and 33 patients, respectively, received BP therapy for more than 3 years. The mean ages of the patients in these groups (BPC, 69.6 \pm 9.1 years; BPDC, 69.7 \pm 9.4 years) were not significantly different $(P=0.924)$. Patients in the BPDC group did not receive BP therapy during the $28.1 \pm 30.6$ months prior to tooth extraction. The BP-administration method did not differ significantly between the 77 patients in the BPC group who received oral $\mathrm{BP}$ and the 28 patients in the BPDC group who received oral alendronate sodium $(P=0.970)$. The mean duration of the medication regimen did not significantly differ $(P=0.057)$ between the patients in the BPC and BPDC groups (65.2 \pm 34.6 months and 53.3 \pm 12.2 months, respectively).

The number of teeth extracted from the 179 patients in the BPC group totaled 537 (306 and 231 from the maxilla and 
Table 1. Characteristics and incidence of post-extraction MRONJ according to BP discontinuation ( $n=465$ patients)

\begin{tabular}{|c|c|c|c|}
\hline \multirow{2}{*}{ Variable } & \multicolumn{2}{|c|}{ Group } & \multirow{2}{*}{$P$-value } \\
\hline & $\mathrm{BPC}$ & BPDC & \\
\hline All patients & $(n=179)$ & $(\mathrm{n}=286)$ & \\
\hline Sex (female:male) & $159: 20$ & $261: 25$ & 0.422 \\
\hline Age $(y r)$ & $70.3 \pm 9.2$ & $67.8 \pm 8.9$ & $0.003 *$ \\
\hline Duration of BP administration (mo) & $40.0 \pm 35.6$ & $14.0 \pm 16.8$ & $<0.001 *$ \\
\hline Duration of IV BP administration (mo) & $23.0 \pm 18.7$ & $13.4 \pm 18.6$ & 0.171 \\
\hline Duration of BP discontinuation (mo) & 0 & $39.0 \pm 35.5$ & $<0.001 *$ \\
\hline PO:PO+IV:IV & $149: 15: 15$ & $260: 15: 11$ & $0.040^{*}$ \\
\hline Surgical extraction with osteotomy & 40 & 61 & 0.818 \\
\hline Osteoporosis:cancer & $175: 3$ & $283: 3$ & 0.680 \\
\hline Post-extraction MRONJ & 1 & 0 & 0.385 \\
\hline $\mathrm{BP}$ administration for more than $3 \mathrm{yr}$ & $(\mathrm{n}=89)$ & $(n=33)$ & \\
\hline Sex (female:male) & $85: 4$ & $32: 1$ & 0.999 \\
\hline Age (yr) & $69.6 \pm 9.1$ & $69.7 \pm 9.4$ & 0.924 \\
\hline Duration of BP administration (mo) & $65.2 \pm 34.6$ & $53.3 \pm 12.2$ & 0.057 \\
\hline Duration of BP discontinuation (mo) & 0 & $28.1 \pm 30.6$ & $<0.001 *$ \\
\hline PO:PO+IV:IV & $77: 5: 7$ & $28: 2: 3$ & 0.970 \\
\hline Surgical extraction with osteotomy & 23 & 10 & 0.650 \\
\hline Osteoporosis:cancer & 89:0 & $30: 3$ & $0.018 *$ \\
\hline Post-extraction MRONJ & 1 & 0 & 0.999 \\
\hline
\end{tabular}

(MRONJ: medication-related osteonecrosis of the jaw, BP: bisphosphonate, BPC: BP-continuation, BPDC: BP-discontinuation, IV: intravenous, PO: oral administration)

$* P<0.05$.

Values are presented as number of patients only or mean \pm standard deviation.

Sang-Hoon Kang et al: The effect of bisphosphonate discontinuation on the incidence of postoperative medication-related osteonecrosis of the jaw after tooth extraction. J Korean Assoc Oral Maxillofac Surg 2020

mandible, respectively). Overall, the extracted teeth were composed of 125 incisors, 180 canines and premolars, and 232 molars. Of the 179 patients, 40 underwent surgical extractions accompanied by alveolar bone removal.

The number of teeth extracted from the 286 patients in the BPDC group was 786 (434 and 352 from the maxilla and mandible, respectively). Overall, the extracted teeth were composed of 173 incisors, 279 canines and premolars, and 334 molars. Of the 286 patients, 61 underwent surgical extractions accompanied by alveolar bone removal.

The BPC and BPDC groups included 175 and 283 patients, respectively, who received BP therapy for the treatment of osteoporosis. In both groups, three patients with cancer received IV injections of ibandronate.

Of the 465 patients included in the study, only one patient in the BPC group developed MRONJ after tooth extraction, while none of the patients in the BPDC group developed MRONJ. The incidence of MRONJ was not significantly different between the two groups $(P=0.385)$.

The patient with MRONJ was a 79-year-old woman with a history of hypertension, but not diabetes, who had received IV injections of ibandronate for 50 months. She had undergone left mandibular second premolar extractions twice to remove remaining dental roots in the same area at the local dental clinic before being admitted to our hospital with a re- sidual root at the site of the extracted tooth. After surgical extraction of the residual root, the patient developed MRONJ. The patient subsequently underwent maxillary incisor and premolar extractions to remove a torus on the lingual side of the left mandible without developing MRONJ.

\section{Discussion}

In the present study, only 1 patient from among the cohort of 465 patients developed MRONJ after tooth extraction. This patient received ibandronate for over 4 years and had already undergone two extraction procedures before visiting our hospital. Therefore, the trauma of having undergone three extraction procedures for the complete removal of the root at the same site may account for her development of MRONJ. This case indicates that although tooth extractions may increase the risk of MRONJ onset, careful tooth extractions will minimize the incidence of post-extraction MRONJ.

The one patient with post-extraction MRONJ was part of the group of 179 patients who underwent tooth extractions without discontinuing BP therapy, as well as a subgroup of 122 patients ( 89 of whom did not discontinue BP medication before tooth extraction) who received BP therapy for more than 3 years. Enforcing BP drug holidays for all patients before tooth extractions should be carefully considered. 
It has been reported that BPs may been retained in the bone due to their extended half-life. Therefore, the discontinuation of BP therapy for several months (a drug holiday) will have little effect on the BPs already incorporated into the bone in cases of tooth extraction procedures ${ }^{16}$. A previous study recommended that necessary tooth extractions should be completed before starting BP therapy and that tooth extraction procedures during BP therapy should be avoided ${ }^{17}$. However, excessive delays in extracting an inflamed tooth may increase the risk of MRONJ, so tooth extractions cannot be completely avoided.

Since no previous study has assessed the incidence of MRONJ following the long-term discontinuation of BP medication prior to extraction procedures, the literature lacks data on extraction socket evaluations to which the results of the present study can be compared. However, a prior report found no evidence of the effect of a short-term oral-BP drug holiday before tooth extraction on reducing the risk of developing MRONJ after tooth extraction and recommended a minimally traumatic extraction technique to lessen the risk of postoperative MRONJ ${ }^{12}$.

Since our study included a limited number of patients $(\mathrm{n}=465)$, additional studies that include more patients from more institutions should be performed to validate our findings concerning the effects of long-term BP discontinuation ( $>3$ months) on the various types of BP therapy according to medication type and period of use. The mean age of the BPC group was significantly higher than the BPDC group. This means that the compared study group was not homogeneous. The current study is a retrospective study which has incomplete medical records for BP therapy. Therefore, a prospective study with clear BP therapy medical records will be helpful for defining the BP drug holiday for dental procedures including tooth extractions.

This study is valuable for the evaluation of the extraction area. Among the patients who were excluded from this study, one patient was diagnosed with MRONJ at the time of tooth extraction. Despite not being diagnosed with MRONJ before the extraction, this patient exhibited a necrotic alveolar bone lesion in the extraction socket at the time of extraction.(Fig. 1) Histopathological results indicated that the lesion was necrotic alveolar bone, suggesting that the patient had already developed MRONJ prior to the tooth extraction procedure.

However, we confused the staging of this case. No clinical evidence, including radiographic support, for necrotic bone was found before the tooth extraction. During the extraction procedure, the necrotic bone of the tooth extraction socket

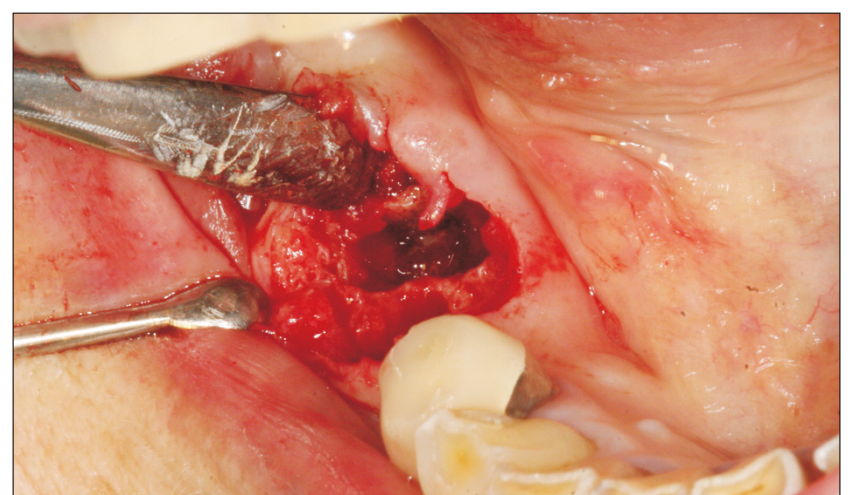

Fig. 1. E.g., Bony necrosis of the extraction socket immediately following tooth extraction. In this case, medication-related osteonecrosis of the jaw may have already developed before the extraction.

Sang-Hoon Kang et al: The effect of bisphosphonate discontinuation on the incidence of postoperative medication-related osteonecrosis of the jaw after tooth extraction. J Korean Assoc Oral Maxillofac Surg 2020

was exposed, and the bony exposure did not last longer than 8 weeks ${ }^{9}$. Therefore, this case may not have been MRONJ. If it was, it would be difficult to determine the stage of the condition. As a result, we suggest a new category of MRONJ with a definition encompassing bony necrosis found in the extraction socket.

A lack of thorough evaluation of the socket at the time of extraction may lead to a misdiagnosis. This may result in false-positive cases of post-extraction MRONJ for cases in which MRONJ had already occurred at the planned extraction area before tooth extraction. Patients diagnosed with post-extraction MRONJ do not always receive a clear preoperative diagnostic evaluation at the time of extraction. Therefore, previous studies suggesting that tooth extraction procedures induce a high rate of post-extraction MRONJ should be assessed carefully to ensure that pre-extraction evaluations of the patients were adequately performed.

Furthermore, it should be reconsidered whether tooth extraction procedures constitute a major risk factor in the development of MRONJ. Future studies with a thorough assessment of whether MRONJ already presents at the planned tooth extraction site are warranted. Understanding the indications of tooth extraction before procedures are performed is essential. Not only should the possible onset of MRONJ before the extraction procedure be considered carefully, but surgeons must also be prepared to treat the possible onset of MRONJ during the extraction procedure because of the possibility of pre-existing MRONJ in the extraction socket area.

Clinicians should consider the following contents for tooth extractions associated with BP therapy. Preventive dental 
treatment should be conducted to preclude the need for invasive dental treatment and BP therapy ${ }^{6}$. The incidence of MRONJ after tooth extraction is relatively low among patients exposed to oral BPs ${ }^{10,11}$, and a short-term drug holiday may not effectively decrease the incidence of MRONJ after tooth extraction ${ }^{12,13}$. In addition, excessive delays in extracting an inflamed tooth may increase the risk of MRONJ, so tooth extractions cannot be completely avoided. The treatment of pre-existing MRONJ in the extraction socket area must be always prepared for tooth extraction-associated BP therapy. Finally, in the tooth extractions associated with BP, a risk evaluation is essential, a proposed algorithm is helpful, and informed consent is necessary ${ }^{18}$.

\section{Conclusion}

The findings of the present study suggest that the accurate diagnosis of MRONJ at the time of extraction is crucial, and that an additional category of MRONJ that encompasses cases of bony necrosis found in the extraction socket during tooth extraction procedures is needed. Our study further indicates that the routine discontinuation of BP medication several months prior to the extraction procedure should be considered carefully.

Lastly, it is difficult to confirm that tooth extractions in patients treated with BP medications for extended periods of time increases their risk of MRONJ. Despite the limitations of our study, we conclude that discontinuing BP medication regimens before tooth extraction is not recommended to reduce the risk of post-extraction MRONJ.

\section{ORCID}

Sang-Hoon Kang, https://orcid.org/0000-0003-3335-3040

Se-Jin Park, https://orcid.org/0000-0003-2206-805X

Moon-Key Kim, https://orcid.org/0000-0002-3634-3705

\section{Authors' Contributions}

S.H.K. obtained data and wrote the manuscript. S.H.K., S.J.P., and M.K.K. drafted the manuscript. M.K.K. participated in article design, coordination, and carefully reviewed and revised the manuscript. All authors read and approved the final manuscript.

\section{Acknowledgements}

This work was supported by the Clinical Research Fund of the National Health Insurance Service Hospital (NHIMC2018CR016).

\section{Ethics Approval and Consent to Participate}

This retrospective study was approved by the Institutional Review Board of the National Health Insurance Service Ilsan Hospital (NHIMC 2018-01-040). Due to the retrospective nature of this study which did not use personal identifying information, informed consent was waived.

\section{Conflict of Interest}

No potential conflict of interest relevant to this article was reported.

\section{References}

1. Otto S, Pautke C, Van den Wyngaert T, Niepel D, Schiødt M. Medication-related osteonecrosis of the jaw: prevention, diagnosis and management in patients with cancer and bone metastases. Cancer Treat Rev 2018;69:177-87.

2. Filleul O, Crompot E, Saussez S. Bisphosphonate-induced osteonecrosis of the jaw: a review of 2,400 patient cases. J Cancer Res Clin Oncol 2010;136:1117-24.

3. Mozzati M, Arata V, Gallesio G, Carossa S. Tooth extraction and oral bisphosphonates: comparison of different surgical protocols. Joint Bone Spine 2011;78:647-8.

4. Kim JW, Baik J, Jeon JH. Dental implant treatment after healing of bisphosphonate-related osteonecrosis of the jaw (BRONJ) in the same region: a case report. J Korean Assoc Oral Maxillofac Surg 2016;42:157-61.

5. Yoneda T, Hagino H, Sugimoto T, Ohta H, Takahashi S, Soen S, et al. Bisphosphonate-related osteonecrosis of the jaw: position paper from the Allied Task Force Committee of Japanese Society for Bone and Mineral Research, Japan Osteoporosis Society, Japanese Society of Periodontology, Japanese Society for Oral and Maxillofacial Radiology, and Japanese Society of Oral and Maxillofacial Surgeons. J Bone Miner Metab 2010;28:365-83.

6. Mücke T, Koerdt S, Jung M, Mitchell DA, Wolff KD, Kesting MR, et al. The role of mylohyoid flap in the treatment of bisphosphonate-related osteonecrosis of the jaws. J Craniomaxillofac Surg 2016;44:369-73.

7. Barba-Recreo P, Del Castillo Pardo de Vera JL, Georgiev-Hristov T, Ruiz Bravo-Burguillos E, Abarrategi A, Burgueño M, et al. Adipose-derived stem cells and platelet-rich plasma for preventive treatment of bisphosphonate-related osteonecrosis of the jaw in a murine model. J Craniomaxillofac Surg 2015;43:1161-8.

8. Damm DD, Jones DM. Bisphosphonate-related osteonecrosis of the jaws: a potential alternative to drug holidays. Gen Dent 2013;61:33-8.

9. Ruggiero SL, Dodson TB, Fantasia J, Goodday R, Aghaloo T, Mehrotra B, et al. American Association of Oral and Maxillofacial Surgeons position paper on medication-related osteonecrosis of the 
jaw--2014 update. J Oral Maxillofac Surg 2014;72:1938-56.

10. Mavrokokki T, Cheng A, Stein B, Goss A. Nature and frequency of bisphosphonate-associated osteonecrosis of the jaws in Australia. $\mathrm{J}$ Oral Maxillofac Surg 2007;65:415-23.

11. Gaudin E, Seidel L, Bacevic M, Rompen E, Lambert F. Occurrence and risk indicators of medication-related osteonecrosis of the jaw after dental extraction: a systematic review and meta-analysis. J Clin Periodontol 2015;42:922-32.

12. Hasegawa T, Kawakita A, Ueda N, Funahara R, Tachibana A, Kobayashi M, et al. A multicenter retrospective study of the risk factors associated with medication-related osteonecrosis of the jaw after tooth extraction in patients receiving oral bisphosphonate therapy: can primary wound closure and a drug holiday really prevent MRONJ? Osteoporos Int 2017;28:2465-73.

13. Otto S, Tröltzsch M, Jambrovic V, Panya S, Probst F, Ristow O, et al. Tooth extraction in patients receiving oral or intravenous bisphosphonate administration: a trigger for BRONJ development? J Craniomaxillofac Surg 2015;43:847-54.

14. Shudo A, Kishimoto H, Takaoka K, Noguchi K. Long-term oral bisphosphonates delay healing after tooth extraction: a single institutional prospective study. Osteoporos Int 2018;29:2315-21.

15. Gatti D, Adami S, Viapiana O, Rossini M. The use of bisphosphonates in women: when to use and when to stop. Expert Opin Pharmacother 2015;16:2409-21.
16. Vescovi P, Campisi G, Fusco V, Mergoni G, Manfredi M, Merigo E, et al. Surgery-triggered and non surgery-triggered Bisphosphonate-related Osteonecrosis of the Jaws (BRONJ): a retrospective analysis of 567 cases in an Italian multicenter study. Oral Oncol 2011;47:191-4.

17. Ensrud KE, Barrett-Connor EL, Schwartz A, Santora AC, Bauer DC, Suryawanshi S, et al. Randomized trial of effect of alendronate continuation versus discontinuation in women with low BMD: results from the Fracture Intervention Trial long-term extension. J Bone Miner Res 2004;19:1259-69.

18. Malden N, Beltes C, Lopes V. Dental extractions and bisphosphonates: the assessment, consent and management, a proposed algorithm. Br Dent J 2009;206:93-8.

How to cite this article: Kang SH, Park SJ, Kim MK. The effect of bisphosphonate discontinuation on the incidence of postoperative medication-related osteonecrosis of the jaw after tooth extraction. J Korean Assoc Oral Maxillofac Surg 2020;46:78-83. https:// doi.org/10.5125/jkaoms.2020.46.1.78 